\title{
Effects of Motor Imagery Combined with Repetitive Task Practice on Sitting Balance of Hemiplegic Patients
}

\author{
Mariko Saito, MS, PT ${ }^{1,2)}$, Tadayoshi Asaka, PhD, PT ${ }^{1)}$, Junko Fukushima, MD, PhD ${ }^{1)}$ \\ 1) Department of Rehabilitation Science, Faculty of Health Sciences, Hokkaido University: West 5 \\ North12, Kita-ku, Sapporo 060-0812, Japan. TEL: +81 11-706-3329, FAX: +81 11-706-3329, \\ E-mail:jf002@hs.hokudai.ac.jp \\ 2) Department of Physical Therapy, Teishin-kai Hospital
}

\begin{abstract}
Purpose] We examined the effects of motor imagery on the sitting balance of 16 hemiplegic patients. [Subjects] The subjects were 6 persons in an intervention group and 10 persons in a non-intervention group. [Methods] All patients received pre- and post-training assessments on the 1st and 5th day. The intervention patients received motor imagery combined with repetitive task practice for 3 consecutive days, while the non-intervention group received no training other than the assessments. With the aid of visual and auditory information the intervention group were instructed to sit and perform imaging as if they flexed their trunk forward and bent their trunk laterally. After motor imagery, they were asked to perform the same repetitive task practice as motor imagery. Elevation and roll angles of the spinal process of L1 and sitting pressure were measured. These parameters were compared between pre- and post-training, and between the intervention and non-intervention groups using ANOVA. [Results] Sitting pressure on the affected side at rest was significantly increased after training, but during forward-flexion, the patients inclined more to the unaffected side, especially left hemiplegic patients. The angles of movement were significantly increased while bending laterally to the affected side. However, sitting pressure showed no significant increase on the affected side. [Conclusion] Short-term motor imagery with repetitive task practice had limited effect on the sitting balance of hemiplegic patients.
\end{abstract}

Key words: Motor imagery, Sitting pressure, Hemiplegic patients

(This article was submitted Sep. 5, 2012, and was accepted Oct. 19, 2012)

\section{INTRODUCTION}

Stroke is one of the major causes of death in Japan and post-stroke hemiplegic patients require long-term care which is administered by family and health workers. Active involvement of the patients in the post-stroke rehabilitation program is crucial if they are to re-acquire independent activities of daily living (ADL). It is important for patients to be active from waking up until bedtime, by eating, taking a bath, toileting and walking. Good sitting balance is essential because patients have to perform many actions while seated. Sandin et al. reported that independence in ADL improved with improvement in sitting balance ${ }^{1)}$. They also stated that patients who showed improved sitting balance while in hospital also achieved greater independence in ADL after discharge ${ }^{1)}$.

Proper sitting balance is symmetrical sitting pressure on the right and left buttocks at rest, ability to transfer sitting pressure in the direction of movement, and maintenance of stable posture during the movements. Center of mass (COM) should be located within the center of the base of support, because postural stability is defined as the ability to control the center of mass (COM) of the head and trunk within the base of support. Sitting pressure should be distributed symmetrically on the ischial tuberosity, and should be within the base of sitting support in order to maintain postural stability while moving in any direction.

Sitting balance training in a rehabilitation program aims at reacquisition of proper sitting balance. Deans et al. showed that patients who received early sitting training within two weeks after stroke onset increased their reaching distance and vertical force on the affected foot while standing ${ }^{2}$. On the other hand, $\mathrm{Au}$-Yeung reported that lateral bending using the Bobath approach failed to improve resting symmetrical sitting pressure ${ }^{3)}$. Mudie et al. divided stroke patients into 4 groups (patients who received visual feedback training, patients who received reaching training for the non-paretic hand, patients who received Bobath training, and patients who received no training), to compare the training effects. They showed that the Bobath approach delivered the greatest improvement just after training, but the group with visual feedback displayed continuous improvement in symmetrical sitting and standing pressure during an observation period lasting 12 weeks ${ }^{4}$.

Recently, training with motor imagery, consisting of imaging as if the subjects moved their body, has been used for rehabilitation of stroke patients. Page et al. examined the effects of motor imagery on the reaching distance of 
Table 1. Demographic features of the subjects in the intervention and non-intervention groups

\begin{tabular}{|c|c|c|c|c|c|}
\hline & & \multicolumn{2}{|c|}{ Intervention $(\mathrm{n}=6)$} & \multicolumn{2}{|c|}{ Non-intervention $(\mathrm{n}=10)$} \\
\hline & & L4 & $\mathrm{R} 2$ & L7 & R3 \\
\hline \multicolumn{2}{|l|}{ gender } & Men 3 & Women 3 & Men 6 & Women 4 \\
\hline \multicolumn{2}{|c|}{ age (years) } & \multicolumn{2}{|c|}{$68.7 \pm 9.1$} & \multicolumn{2}{|c|}{$73.2 \pm 8.5$} \\
\hline \multicolumn{2}{|c|}{ hight (cm) } & \multicolumn{2}{|c|}{$159.4 \pm 5.7$} & \multicolumn{2}{|c|}{$161.9 \pm 6.8$} \\
\hline \multicolumn{2}{|c|}{ weight (kg) } & \multicolumn{2}{|c|}{$63.6 \pm 17.6$} & \multicolumn{2}{|c|}{$60.4 \pm 6.7$} \\
\hline \multicolumn{2}{|c|}{ BMI } & \multicolumn{2}{|c|}{$24.8 \pm 5.8$} & \multicolumn{2}{|c|}{$23 \pm 2.2$} \\
\hline \multicolumn{2}{|c|}{ time from onset (month) } & \multicolumn{2}{|c|}{$38.5 \pm 49.8$} & \multicolumn{2}{|c|}{$63.5 \pm 65.9$} \\
\hline \multirow{2}{*}{ type } & infarction & \multicolumn{2}{|c|}{2} & \multicolumn{2}{|c|}{6} \\
\hline & heamorrhage & \multicolumn{2}{|l|}{4} & \multicolumn{2}{|l|}{4} \\
\hline \multirow{2}{*}{ Br-stage } & upper limb & \multicolumn{2}{|c|}{$3.3 \pm 0.9$} & \multicolumn{2}{|c|}{$4.1 \pm 1$} \\
\hline & lower limb & \multicolumn{2}{|c|}{$3.8 \pm 0.7$} & \multicolumn{2}{|c|}{$4.3 \pm 0.8$} \\
\hline \multicolumn{2}{|l|}{ HDS-R } & \multicolumn{2}{|c|}{$27.3 \pm 2$} & \multicolumn{2}{|c|}{$25.6 \pm 2.9$} \\
\hline \multicolumn{2}{|c|}{ Barthel Index } & \multicolumn{2}{|c|}{$67.5 \pm 23.8$} & $80.5 \pm$ & $=11.3$ \\
\hline FIM & & $98.3 \pm$ & & $113.5 \pm$ & $=7.6$ \\
\hline FIM (m & & $64.8 \pm$ & 19.4 & $80.6 \pm$ & $6.6^{*}$ \\
\hline hemiaso & & 1 & & 1 & \\
\hline hemispa & & 1 & & 1 & \\
\hline & & Intervent & ion group & Non-interve & ention group \\
\hline & & L- hemiplegia & R-hemiplegia & L-hemiplegia & R-hemiplegia \\
\hline Brsto & upper limb & $2.8 \pm 0.4$ & $4.5 \pm 0.5$ & $3.7 \pm 0.9$ & $5 \pm 0.8$ \\
\hline Br-stage & lower limb & $3.5 \pm 0.5$ & $4.5 \pm 0.5$ & $4.1 \pm 0.8$ & $4.7 \pm 0.5$ \\
\hline HDS-R & & $26.5 \pm 1.8$ & $29 \pm 1$ & $25 \pm 2.7$ & $27 \pm 2.8$ \\
\hline Barthel & & $51.3 \pm 7.4$ & $100 \pm 0$ & $77.1 \pm 11.3$ & $88.3 \pm 6.2$ \\
\hline FIM & & $85.5 \pm 10.2$ & $124 \pm 0$ & $110 \pm 6.5$ & $121.7 \pm 0.9$ \\
\hline FIM (m & & $52 \pm 8.5$ & $90.5 \pm 0.5$ & $77.9 \pm 6.1$ & $87 \pm 0.8$ \\
\hline
\end{tabular}

Average \pm standard deviation (SD) is shown. Significant differences are shown by asterisks. L: left hemiplegia, R: right hemiplegia, Br-stage: Brunnstrom stage, HDS-R: Hasegawa Dementia Scale Revised, FIM: Functional Independence Measure

the upper limb to an object, and reported that the patients not only showed improvement in reaching movement, but also in other $\mathrm{ADL}^{5}$ ). Dunsky et al. reported the effect of motor imagery on gait ${ }^{6}$. Although it is difficult to confirm whether the patients are performing motor imagery properly, Dunsky et al. and Malouin et al. used auditory stimulation, a metronome and a therapist's explanation about the movements during motor imaging, ${ }^{6,7)}$. Moreover, as Feltz reported, it is more effective to perform the same movement of the motor imagery after imaging, compared to motor imagery alone ${ }^{8)}$. For stroke patients, Barclay-Goddard et al. reported that motor imagery of the upper limb combined with motor training was effective for movement recovery ${ }^{9}$. Molouin et al. showed that training stroke patients with imagery and movements was most effective for standing up and sitting down ${ }^{10}$. These reports relate to the effects of motor imagery with repetitive task practice on the recovery of stroke patients. However, there has been no report regarding the effects of motor imagery training on sitting balance. Therefore, the purpose of this study was to determine the effects of motor imagery training combined with repetitive task practice on hemiplegic patients.

\section{SUBJECTS AND METHODS}

\section{Subjects}

We studied 16 stroke patients (11 left and 5 right hemiplegics) who were enrolled in the rehabilitation program at the Teishin-kai Hospital and Rapas Nursing Home rehabilitation facilities in Sapporo. The patients in this study were: able to sit independently without back support for at least 1 minute; free from serious disorders of hearing and language understanding, and had scores greater than 20 on the Hasegawa Dementia Scale Revised (HDS-R); and were able to understand the task and cooperate in the experiment. Before the experiment, all patients in the intervention and non-intervention groups received regular conventional physical therapy. None of the subjects received sitting balance training with visual feedback during regular physical therapy. All patients provided their informed consent to the procedures which were approved by the ethics committee of the Hokkaido University Faculty of Health Sciences.

The sixteen subjects were randomly divided into two groups. The intervention group consisted of 6 patients $(3$ men, 3 women) who performed motor imagery training with visual feedback plus repetitive movement practice. The 
other 10 subjects formed a non-intervention group ( 6 men, 4 women) who received no training other than routine conventional physical therapy. In the intervention group, the mean age was $68.7( \pm 9.1 \mathrm{SD})$ years, average height was 159.4 $( \pm 5.7 \mathrm{SD}) \mathrm{cm}$, and average weight was $63.6( \pm 17.6 \mathrm{SD}) \mathrm{kg}$. In the non-intervention group, the mean age was $73.2( \pm 8.5$ SD) years, average height was $161.9( \pm 6.8 \mathrm{SD}) \mathrm{cm}$, and average weight was $60.4( \pm 6.7 \mathrm{SD}) \mathrm{kg}$. Time since the onset of stroke was $38.5( \pm 49.8 \mathrm{SD})$ months in the intervention group, and 63.5 ( $\pm 65.9 \mathrm{SD})$ months, in the non-intervention group. One subject in the non-intervention group showed left hemiasomatognosia, and another showed left side visuospatial neglect. One patient in the intervention group showed both left hemiasomatognosia and left side visuo-spatial neglect. Average Brunnstrom stage was $3.3( \pm 0.9 \mathrm{SD})$ for the upper limbs and $3.8( \pm 0.7 \mathrm{SD})$ for the lower limbs in the intervention group, and $4.1( \pm 1.0 \mathrm{SD})$ and $4.3( \pm 0.8$ $\mathrm{SD})$, respectively, in the non-intervention group. Average Barthel Indexes were $67.5( \pm 23.8 \mathrm{SD})$ in the intervention group, and 80.5 ( $\pm 11.3 \mathrm{SD})$ in the non-intervention group. All subjects in the intervention group and 5 subjects of the non-intervention group had sensory disturbances. Average functional independence measures (FIM) were $98.3( \pm 20.0$ $\mathrm{SD})$ and $113.5( \pm 7.6 \mathrm{SD})$ in the intervention and non-intervention groups, respectively. No significant differences were found between the two groups in homogeneity except for the movement subscale of the FIM, which was significantly lower in the intervention group. The demographic findings are shown in Table. 1

Assessments were done on the 1 st and the 5 th day, for both groups. The assessment procedure was as follows. Subjects were required to sit on a stool without a back support. The height of the stool was adjusted so that their knee angle was $90^{\circ}$ and their feet were flat on the floor during sitting. A computer display showed their sitting pressure using several colors, as shown in Figure 1. First, subjects were instructed to sit without back support and to watch the display showing their sitting pressure, and try to adjust their posture, so that left and right sitting pressure became symmetric (at rest). Sitting pressure and the elevation angle, which is defined as the angle from the horizontal plane (x-axis) of L1, were measured for each subject during bending forward, as well as the roll angle of L1 for lateral bending. Next, the subjects looked at photographs depicting a healthy young subject who was bending the thorax on the pelvis through a range of motions according to the click of the metronome (30 bpm). On the 2nd, 3rd and 4th days, only the intervention group patients performed motor imagery and executed the repetitive task practice training, while the non-intervention group received no training. The intervention group training was as follows. The patients were asked to imagine trunk flexion, as if they bent themselves forward in time with a metronome. They were instructed "to imagine" but not to actually move any part of the body. To make them concentrate on the task and for encouragement, one of the examiners shouted 'one, two, three, four', pointing to a demonstration picture. The other examiners carefully observed the subjects' condition, and later we confirmed their performances on videos. After "imaging", subjects were asked to execute the same

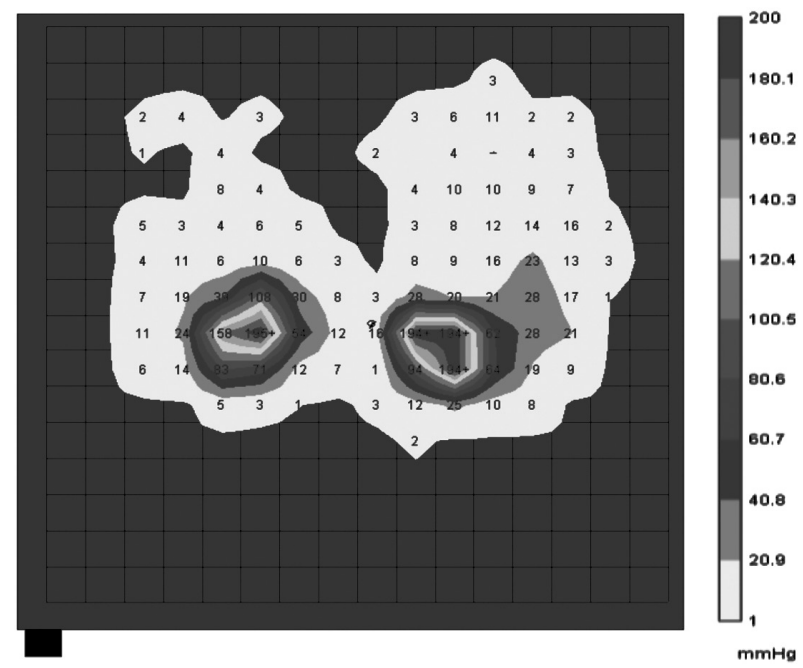

Fig. 1. An example of sitting pressure at rest in a left hemiplegic. Up indicates anterior. The small figures indicate sitting pressure $(\mathrm{mmHg})$.

movement as imagery, aided by identical visual and auditory stimulation, as in the assessment. Then, they were instructed to bend themselves laterally, alternating between right and left lateral bending movements according to the click of a metronome $(30 \mathrm{bpm})$. Lateral bends were executed with visual information 7 times, after subjects had imagined their own lateral bending movements. The total time of the program was 20 minutes.

Sitting pressure was measured with the Force Sensitive Application version 4.0 (FSA 4.0) (Takano Inc., Nagano). The system consists of a thin mat with 256 strain gauge sensor units. The data were sampled at a frequency of $5 \mathrm{~Hz}$. The sitting pressure was continuously displayed on the monitor to both the experimenters and the subjects. A sensor was put on the skin surface of the 1 st lumbar process and sacral vertebrae. Elevation and roll angles of L1 were recorded by 3-Space ISOTRAK (Polhemus Inc., Colchester) during forward flexing and lateral bending. Position (x, y and $\mathrm{z}$ coordinates) was recorded by 3 -Space ISOTRAK at a sampling frequency of $12.5 \mathrm{~Hz}$. The analysis was performed for all subjects. For measuring sitting pressure, each value of the affected and unaffected side was divided by the sum of the bilateral sitting pressures. To show the involvement of affected and unaffected sides, we calculated the proportion of the sitting pressure on each side. If the pressure were symmetric, each side would be $50 \%$.

The degree of forward flexion was indicated by the elevation angle, and lateral bending was shown by the roll angle of the 1st lumbar vertebrae. The paired t-test was used to evaluate the differences between pre- and post-intervention. Analysis of variance (ANOVA) was used to detect the effects of intervention for time (pre- vs. post training) and group (intervention vs. non-intervention), in order to examine the effects of training. The level of significance was chosen as $95 \%$ for all statistical analyses. 
Table 2. Comparison of the results of the intervention and non-intervention groups, pre- and post-intervention

\begin{tabular}{|c|c|c|c|c|c|}
\hline \multirow{2}{*}{ A. sitting pressure (\%) } & \multicolumn{2}{|c|}{ Intervention group } & \multicolumn{2}{|c|}{ Non-intervention group } & \multirow{2}{*}{$\begin{array}{l}\text { ANOVA } \\
\mathrm{F} \\
\end{array}$} \\
\hline & pre & post & pre & post & \\
\hline at rest (affected side) & $43 \pm 7$ & $50 \pm 7 *$ & $44 \pm 9$ & $4 \pm 4$ & $\mathrm{~F}(620,1)=21.35^{*}$ \\
\hline flexion forward(affected side) & $43 \pm 13$ & $33 \pm 17 *$ & $48 \pm 14$ & $48 \pm 9$ & $\mathrm{~F}(318,1)=9.26^{*}$ \\
\hline bending to affected side & $81 \pm 20$ & $87 \pm 20$ & $75 \pm 21$ & $83 \pm 17$ & $F(169,1)=0.22$ \\
\hline bending to unaffected side & $9 \pm 6$ & $2 \pm 3^{*}$ & $16 \pm 14$ & $14 \pm 16$ & $F(165,1)=0.22$ \\
\hline \multirow[t]{2}{*}{ B, flexing or bending angles (degree) } & \multicolumn{2}{|c|}{ Intervention group } & \multicolumn{2}{|c|}{ Non-intervention group } & ANOVA \\
\hline & pre & post & pre & post & $\mathrm{F}$ \\
\hline flexion forward (affected side) & $29 \pm 21$ & $36 \pm 18$ & $38 \pm 24$ & $39 \pm 19$ & $\mathrm{~F}(331,1)=0.77$ \\
\hline bending to affected side & $9 \pm 3$ & $19 \pm 9^{*}$ & $12 \pm 9$ & $11 \pm 7$ & $\mathrm{~F}(162,1)=18.98^{*}$ \\
\hline bending to unaffected side & $10 \pm 3$ & $18 \pm 9^{*}$ & $17 \pm 18$ & $12 \pm 7^{*}$ & $\mathrm{~F}(169,1)=11.28^{*}$ \\
\hline \multirow[t]{2}{*}{ C. sitting pressure in right hemiplegics (\%) } & \multicolumn{2}{|c|}{ Intervention group } & \multicolumn{2}{|c|}{ Non-intervention group } & ANOVA \\
\hline & pre & post & pre & post & $\mathrm{F}$ \\
\hline at rest (affected side) & $47 \pm 6$ & $54 \pm 2^{*}$ & $48 \pm 2$ & $43 \pm 4^{*}$ & $\mathrm{~F}(198,1)=114.22^{*}$ \\
\hline flexion forward (affected side) & $47 \pm 6$ & $48 \pm 6^{*}$ & $59 \pm 13$ & $55 \pm 5^{*}$ & $F(106,1)=3.10$ \\
\hline bending to affected side & $90 \pm 5$ & $99 \pm 1^{*}$ & $88 \pm 15$ & $88 \pm 16$ & $F(49,1)=1.70$ \\
\hline bending to unaffected side & $11 \pm 6$ & $1 \pm 1^{*}$ & $10 \pm 14$ & $9 \pm 13$ & $F(51.1)=1.66$ \\
\hline \multirow[t]{2}{*}{ D.sitting pressure in left hemiplegics (\%) } & \multicolumn{2}{|c|}{ Intervention group } & \multicolumn{2}{|c|}{ Non-intervention group } & ANOVA \\
\hline & pre & post & pre & post & $\mathrm{F}$ \\
\hline at rest (affected side) & $41 \pm 7$ & $48 \pm 10^{*}$ & $42 \pm 10$ & $46 \pm 3^{*}$ & $F(421,1)=3.02$ \\
\hline flexion forward (affected side) & $40 \pm 16$ & $22 \pm 14^{*}$ & $44 \pm 12$ & $45 \pm 9$ & $\mathrm{~F}(209,1)=24.90^{*}$ \\
\hline bending to affected side & $76 \pm 24$ & $80 \pm 22$ & $69 \pm 20$ & $80 \pm 16^{*}$ & $F(113,1)=0.94$ \\
\hline bending to unaffected side & $7 \pm 6$ & $2 \pm 3^{*}$ & $18 \pm 14$ & $17 \pm 16$ & $\mathrm{~F}(111,1)=0.93$ \\
\hline
\end{tabular}

Results of sitting pressure (A) and bending angles (B) for all subjects are shown. (C) and (D) show sitting pressures (\%) of right and left hemiplegics, respectively. Significant differences were found between pre-and post-intervention by the paired t-test. Interaction was evaluated using ANOVA (F-value). Significant differences are shown by asterisks

\section{RESULTS}

The results are summarized in Table 2. At rest, the mean $\pm \mathrm{SD}$ percentages of sitting pressure on the affected side in the intervention and non-intervention groups were $43 \pm 7 \%$, and $44 \pm 9 \%$ before intervention, respectively. There was no significant difference between the two groups $(\mathrm{t}(310)=0.89, \mathrm{p}=0.37)$. Subjects in both groups showed higher pressure on the unaffected side. After training, the intervention group increased sitting pressure to $50 \pm 7 \%$ on the affected side. However, the non-intervention group experienced no significant increase $(45 \pm 4 \%)$. ANOVA showed that there was a significant interaction between intervention and group of the sitting pressure on the affected side at rest $(\mathrm{F}(620,1)=10.33, \mathrm{p}<0.001)$. This indicates that training intervention increased the sitting pressure on the affected side, suggesting that it was effective at achieving symmetrical sitting pressure at rest.

In patients with right hemiplegia ( $\mathrm{C}$ in Table 2$)$, paretic side sitting pressure increased after training intervention ( $47 \pm 6 \%$ vs. $54 \pm 2 \%, p<0.001$ ). The non-intervention group also showed a decrease on the paretic side from $48 \pm 2 \%$ to $43 \pm 4 \%, \mathrm{p}<0.001$ ), and the interaction between intervention and group was significant $(F(198,1)=114.2, p<0.001)$. These results indicate that the training intervention was effective at improving the symmetry of sitting pressure at rest. In patients with left hemiplegia, sitting pressure of the affected side increased from $41 \pm 7 \%$ to $48 \pm 10 \%$ after training intervention, while in the non-intervention patients it increased from $42 \pm 10 \%$ to $46 \pm 3 \%$. These results showed a significant main effect of group $(F(198,1)=84.34, p<0.001)$, but the main effects between before and after intervention ( $F$ $(198,1)=0.05, \mathrm{p}=0.82)$, and interaction were not significant $(\mathrm{F}(421,1)=3.2, \mathrm{p}=0.08)$.

On the affected side, the sitting pressure during forward flexion in the intervention group decreased from $43 \pm 13 \%$ to $33 \pm 17 \%$, while the non-intervention group showed no significant difference between before $(48 \pm 14 \%)$ and after $(48 \pm 9 \%)$ the training. ANOVA revealed a significant interaction $(\mathrm{F}(318,1)=9.3, \mathrm{p}<0.001)$. This indicates that the intervention did not improve the sitting pressure during forward flexion, and that patients tended to load on the non-paretic side during forward flexion after training. The movement angle also tended to decrease after the intervention, from $39 \pm 21^{\circ}$ to $36 \pm 18^{\circ}$, and the non-intervention group tended to show an increase (before $38 \pm 24^{\circ}$, after $39 \pm 19^{\circ}$; however, there was no significant interaction $(\mathrm{F}$ $(331,1)=0.77, p=0.38)$. In right hemiplegic patients, sitting pressure of the paretic side was $47 \pm 6 \%$ before training, and it significantly increased to $48.6 \%$ after training $(\mathrm{p}=0.04)$. On the other hand, the non-intervention group showed a decrease from $59.13 \%$ to $55.5 \%$; however, no interaction was observed between group and intervention $(F(106,1)=3.1$, $\mathrm{p}=0.08)$. 
In left hemiplegic patients, sitting pressure of the paretic side was $40 \pm 16 \%$ before training and $23 \pm 14 \%$ after training, a significant decrease, while the non-intervention group showed no significant difference ( $44 \pm 12 \%, 45 \pm 9 \%$ ). There was a significant interaction between group and intervention, indicating that the patients with left hemiplegia did not load on the paretic side during forward flexion after training.

During lateral flexion to the affected side, the angles of movement increased $9 \pm 3^{\circ}$ to $19 \pm 9^{\circ}(\mathrm{p}<0.001)$ in the intervention group, while the non-intervention group showed no significant change $(\mathrm{p}=0.44)$. There was a significant interaction between group and intervention $(F(162,1)=18.98$, $\mathrm{p}<0.001)$. The sitting pressure tended to increase after training ( $81 \pm 20 \%$ to $87 \pm 20 \%, p=0.09)$ without significance. In the non-intervention group, there was no significant difference $(75 \pm 21 \%$ to $83 \pm 17 \%)$, and no significant interaction was observed $(\mathrm{F}(169.1)=0.22, \mathrm{p}=0.64)$.

In the right hemiplegics, sitting pressure during bending to the affected side significantly increased after training from $90 \pm 5 \%$ to $99 \pm 1 \%(\mathrm{p}<0.001)$. Patients in the non-intervention group showed no significant increase $(88 \pm 15 \%$ to $88 \pm 16 \%, \mathrm{p}=0.95)$, and no significant interaction was observed $(\mathrm{F}(49,1)=1.70, \mathrm{p}=0.20)$. Left hemiplegic patients showed an increase after the training intervention $\left(7 \pm 2^{\circ}\right.$ to $20 \pm 9^{\circ}$ ), and patients in the non-intervention group also increased the angle $\left(9 \pm 8^{\circ}\right.$ to $11 \pm 7^{\circ}$, data not shown). ANOVA revealed that the main effect was significant in intervention $(F(111,1)=12.97, p<0.001)$, with significant interaction $(\mathrm{F}(111,1)=15.65, \mathrm{p}<0.001$, data not shown), indicating the movement angle increased by training intervention. However, the sitting pressures of these patients showed no significant difference $(76 \pm 24 \%$ vs. $80 \pm 22 \%$, $\mathrm{p}=0.86$ ), while the non-intervention group showed a significant increase $(69 \pm 20 \%$ vs. $80 \pm 16 \%, \mathrm{p}<0.001)$. There was no significant interaction $(\mathrm{F}(113,1)=0.94, \mathrm{p}=0.34)$.

During lateral flexion to the unaffected side, movement angle was $10 \pm 3^{\circ}$ before and $18 \pm 9^{\circ}$ after training, a significant increase $(p<0.001)$, in the intervention group. The non-intervention group showed a significant decrease from $17 \pm 18^{\circ}$ to $12 \pm 7^{\circ}(\mathrm{p}=0.004)$. ANOVA revealed a significant interaction $(\mathrm{F} \quad(169,1)=11.28, \mathrm{p}<0.001)$. These results indicate the training intervention was effective at improving the angle of lateral flexion on the unaffected side.

To bend laterally to the unaffected side, the subjects must reduce pressure on the affected side. Sitting pressure on the unaffected side significantly decreased after the training intervention $(9 \pm 6 \%$ vs. $2 \pm 3 \%, p<0.001)$. The non-intervention group showed no significant difference $(16 \pm 14 \%$ vs. $14 \pm 16 \%$, $\mathrm{p}=0.95)$. However, there was no significant interaction $(\mathrm{F}(165,1)=2.02, \mathrm{p}=0.16)$.

In right hemiplegic patients, sitting pressure of the affected side decreased significantly ( $11 \pm 6 \%$ vs. $1 \pm 1 \%, \mathrm{p}<0.001)$, while the non-intervention patients showed no significant difference $(10 \pm 14 \%$ vs. $9 \pm 13 \%, \mathrm{p}=0.23)$. However, there was no significant interaction between group and intervention $(\mathrm{F}(51,1)=1.66, \mathrm{P}=0.20)$. In left hemiplegics, the sitting pressure of the unaffected side during bending to the left was $7 \pm 6 \%$ before training and $2 \pm 3 \%$ after training, a significant decrease $(\mathrm{p}<0.001)$, and the non-intervention group also showed a decrease, but it was not significant $(18 \pm 14 \%$ vs. $17 \pm 16 \%, \mathrm{p}=0.85)$. There was no significant interaction $(\mathrm{F}(111,1)=0.93, \mathrm{p}=0.34)$.

A negative correlation was shown between the time from the onset of stroke and change of sitting pressure in the subjects $(\mathrm{r}=-0.86, \mathrm{p}=0.03)$. There was a positive correlation between the Barthel Index and change in sitting pressure on affected side $(r=0.82, \mathrm{p}=0.045)$.

To summarize the results, the sitting pressure at rest became more symmetric after training, compared to its pre-training distribution suggesting a positive effect of intervention. The angle of lateral bending also increased significantly, and ANOVA showed significant interaction between time and group. In the comparison of patients who suffered right hemiplegia with left hemiplegia, right hemiplegics significantly increased the proportion of sitting pressure at rest on the affected side. On the other hand, left hemiplegics significantly decreased sitting pressure of the affected side during forward flexion. There was a negative correlation between the time from the onset of stroke and changes in sitting pressure on the affected side, and a positive correlation between the Barthel Index and change in sitting pressure on the affected side.

\section{DISCUSSION}

The results of our present study suggest that motor imagery with repetitive task practice has limited, but positive effects on sitting balance. When they flexed forward, the intervention group patients showed lower sitting pressure on the paretic side and higher pressure on the non-paretic side, compared to the patients in the non-intervention group. The reasons why they showed deterioration in sitting weight pressures after training are suggested as follows.

First, the intervention group included left hemiplegic patients who had a lower score in the motor component of the Barthel Index. The reason why left hemiplegic patients were unable to shift sitting pressure to the affected side is that they had to depend on visual feedback, because it was difficult for them to process the spatial information necessary for postural control $^{11)}$. Although there were no patients with Pusher's syndrome in the present study ${ }^{12)}$, left hemiplegic patients have been reported to have more difficulties in postural control than those with right hemiplegia ${ }^{13)}$. Possibly, the tilting body axis of left hemiplegic patients may be corrected by visual feedback. Only 3 left hemiplegic subjects were diagnosed as hemiasomatognosia and hemispatial agnosia by clinical neuropsychological tests. However, we speculate most of the hemiplegic subjects had difficulties in spatial cognition, even if they did not reveal abnormalities in the clinical tests. In this study, when patients performed forward flexion of the trunk, they were unable to use visual feedback to modify their postural axis, because it was difficult for them to see the display during forward flexion. The decrease of sitting pressure on the affected side when the patients flexed forward may have been due to the lower motor function of the intervention group, and the tilting body axis of left hemiplegic patients. The results also suggest that the left 
hemiplegic patients may have received less benefit from the training without visual feedback than the right hemiplegic patients.

In lateral bending, the training increased the angles of both the paretic and non-paretic sides. This indicates improved upper trunk movement above the pelvis. Regarding sitting pressure, however, no significant difference was observed except for a tendency to decrease on the paretic side during bending to the unaffected side. A possible explanation for this is that stroke patients are reportedly unable to move the pelvis as a support, because the activities of the surface muscles, such as the abdominal rectus, and external oblique abdominal muscle, are affected on the paretic side ${ }^{14}$.

This study had several limitations, one being the small sample size and short training period. Although only the intervention group received image training in the present study, ideally, we should have compared three groups, a repetitive task practice training alone group, a motor imagery plus repetitive task practice training group, and a non-intervention group, in order to confirm the exclusive effectiveness of motor imagery.

It is difficult to confirm whether subjects imagine their movement properly or not. Sharma et al. showed with positron emission tomography (PET) of the brain during movement that the supplementary motor area, inferior parietal area contralateral to the movements and the ipsilateral cerebellum were activated ${ }^{15)}$. Page et al. reported activation of the bilateral primary motor, premotor, and ipsilateral parietal areas ${ }^{5)}$. Since these areas are involved in planning, preparing and executing movement, it is possible that motor imagery would enhance them. From a clinical aspect, the effectiveness of training with combined motor imagery and repetitive task practice suggests its clinical application for outpatients in the rehabilitation stage. After stroke patients have learned the procedure in hospital, they would be able to practice it at home. As reported by Page et al. ${ }^{16)}$, motor imagery combined with repetitive task practice showed retention of effects for at least 3 months after the last intervention. Future studies should examine whether retention of motor changes occurred in the present study.

In conclusion, motor imagery training combined with repetitive task practice had a limited effect on the sitting balance of hemiplegic patients.

\section{ACKNOWLEDGEMENT}

We thank Drs. T. Hatta, H. Saito and S. Kasahara for helping with the studies, and we appreciate the English language advice given by Dr. P. Olley. We also appreciate the cooperation of Ms. M. Sawada, a director of Physical Therapy at Teishin-kai Hospital. This work was supported by a Grant-in-Aid for Scientific Research from the Ministry of Education, Culture, Sports, Science and Technology of Japan (2350057701)

\section{REFERENCES}

1) Sandin KJ, Smith BS: The measure of balance in sitting in stroke rehabilitation prognosis. Stroke, 1990, 21: 82-86. [Medline] [CrossRef]

2) Dean CM, Channon EF, Hall JM: Sitting training early after stroke improves sitting ability and quality and carries over to standing up but not to walking: a randomized controlled trial. Aust J Physiother, 2007, 53: 97-102. [Medline] [CrossRef]

3) Au-Yeung SS: Does weight-shifting exercise improve postural symmetry in sitting in people with hemiplegia? Brain Inj, 2003, 17: 789-797. [Medline] [CrossRef]

4) Mudie MH, Winzeler-Mercay U, Radwan S, et al.: Training symmetry of weight distribution after stroke: a randomized controlled pilot study comparing task-related reach, Bobath and feedback training approaches. Clin Rehabil, 2002, 16: 582-592. [Medline] [CrossRef]

5) Page SJ, Szaflarski JP, Eliassen JC, et al.: Cortical plasticity following motor skill learning during mental practice in stroke. Neurorehabil Neural Repair, 2009, 23: 382-388. [Medline]

6) Dunsky A, Dickstein R, Marcovitz E, et al.: Home-based motor imagery training for gait rehabilitation of people with chronic poststroke hemiparesis. Arch Phys Med Rehabil, 2008, 89: 1580-1588. [Medline] [CrossRef]

7) Malouin F, Richards CL, Durand A, et al.: Mental practice for relearning locomotor skills. Phys Ther, 2010, 90: 240-251. [Medline] [CrossRef]

8) Feltz DL, Landers DM: The effects of mental practice on motor skill learning and performance: a meta-analysis. J Sport Psychol, 1983, 5: $25-57$.

9) Barclay-Goddard RE, Stevenson TJ, Poluha W, et al.: Mental practice for treating upper extremity deficits in individuals with hemiparesis after stroke (Review). The Cochrane Database Sys Rev 2011, DOI: 10.1002/14651858.CD005950.pub4.

10) Malouin F, Richards CL, Durand A, et al.: Added value of mental practice combined with a small amount of physical practice on the relearning of rising and sitting post-stroke: a pilot study. J Neurol Phys Ther, 2009, 33: 195-202. [Medline]

11) Spinazzola L, Cubelli R, Sala SD: Impairments of trunk movements following left or right hemisphere lesions: dissociation between apraxic errors and postural instability. Brain, 2003, 126: 2656-2666. [Medline] [CrossRef]

12) Karnath HO, Ferber S, Dichgans J: The origin of controversies pushing: Evidence for a second graviceptive system in humans. Neurology, 2000, 55: 1298-1304. [Medline] [CrossRef]

13) Rode G, Tiliket C, Boisson D.: Predominance of postural imbalance in left hemiparetic patients. Scand J Rehabil Med, 1997, 29: 11-16. [Medline]

14) Dickstein R, Shefi S, Marcovitz E, et al.: Electromyographic activity of voluntarily activated trunk flexor and extensor muscles in post-stroke hemiparetic subjects. Clin Neurophysiol, 2004, 115: 790-796. [Medline] [CrossRef]

15) Sharma N, Pomeroy VM, Baron JC, et al.: Motor imagery: a backdoor to the motor system after stroke? Stroke, 2006, 37: 1941-1952. [Medline] [CrossRef]

16) Page SJ, Murray C, Hermann V, et al.: Retention of motor changes in chronic stroke survivors who were administered mental practice. Arch Phys Med Rehabil, 2011, 92: 1741-1745. [Medline] [CrossRef] 